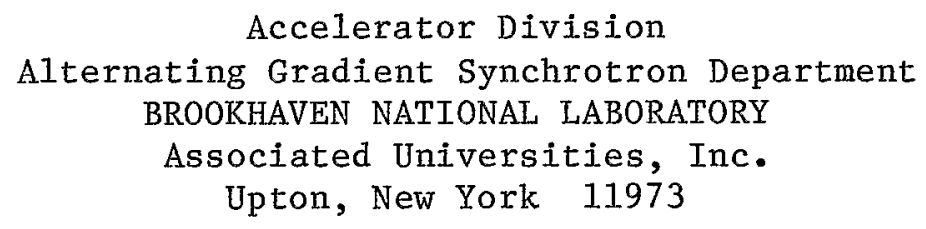

THE MAXIMUM THEORETICAL PRESSURE GRADIENT IN THE AGS

K. Welch and J. Tuzzolo

February 3, 1989 


\title{
THE MAXIMUM THEORETICAL PRESSURE GRADIENT IN THE AGS
}

\author{
Kimo Welch \\ Joseph Tuozzolo
}

February 3, 1989

\section{THE PROBLEM}

The new vacuum I\&C system provides excellent visibility in diagnosing vacuum problems in the AGS. In the process of diagnosing problems, there are instances where indicated pressure differences between adjacent pumps seem inordinately large. This raises several questions: 1) Are they real differences? 2) If they are not real, are they the result of a faulty pump, pump high voltage cable or power supply? 3) What is the theoretical maximum possible pressure gradient? This third question is addressed in this technical note. Only steady-state conditions are considered.

\section{GENERAL CONSIDERATIONS}

There will be a nearly symmetrical pressure gradient in the AGS about some source of gas entering the system. There is a maximum gradient which can exist. This gradient depends on the conductance of the beam chamber, $\mathbf{c}_{C}$, and the speed delivered to the chamber by each pump, $\mathbf{s}_{C}(\mathbf{P})$. The effects of drop-off in pump speed with pressure will in all cases lead to a more gradual pressure gradient between pumps.

There may be slight asymmetries in the pressure gradients on each side of a leak. These can be caused by other smaller leaks. Also, asymmetries will be caused by the presence of "five-foot" straight-sections, rf cavities, etc. These, however, are second order effects.

The results of three cases will be given; Case I: A leak exists an alternating gradient magnet chamber (AGMC) flange; Case II: A leak exists in a pump; Case III: The I/P of the pump nearest a leak is the same as all other pumps, but the speed of that pump is zero because of pump heating.

\section{SPECIFICS OF THE AGS CONFIGURATION}

Contrary to common belief, ${ }^{[1]}$ a vacuum system, under molecular flow conditions, may be completely modeled as a simple, linear, electrical circuit. With such a model, one can solve for the exact value of the collective speed, $\Sigma S$, of all the AGS pumps, produced at any one location in the ring. This requires the construction and manipulation of $120 \times 120$ determinants (i.e., 120 unknowns). Use of matrix theory to make such calculations has been done, ${ }^{[2]}$ but seems a bit esoteric.

When a system gets too unwieldy to conveniently mathematically model, one can resort to modeling the system with resistors and batteries. One of the authors did this at SLAC in 1969 , as a tool for diagnosing vacuum problems in a 100-meter sector of the accelerator. 
The AGS may be modeled as two parallel electrical circuits joined at a "current source", the leak. Pump speed at the flange of any AGMC is simply the sum of $\mathbf{s}_{\mathrm{C}}$ and the results of the pumping of all other AGS pumps through conductances of varying lengths. Because the "circuits" are parallel, we may use a conductance for the chambers, $\mathrm{C}_{\mathrm{C}}$, which is twice that of one AGMC. Also, the pumping speed between any two AGMCs, $\mathbf{s}_{C}$, may be viewed as twice that delivered by any one AGS pump. Define the speed produced at the pump farthest removed from the leak as $\mathbf{s}_{\mathrm{k}}$, speed at the next pump, closer to the leak, as $\mathbf{s}_{\mathrm{k}-1}, \ldots$ Clearly,

$$
s_{k-n-1}=\frac{\left(s_{k-n} s_{C}\right) c_{c}}{s_{k-n}+s_{C}+c_{c}}
$$

Equation (1) converges to 64.66436 for $\mathbf{n} \geq 12$, and this is the value of $\Sigma S$. Remember, this is for an "air leak". The value of $\Sigma \mathbf{S}$ will differ for different gases.

Define $\mathbf{P}_{0}$ as the pressure indicated by the pump at the leak, $\mathbf{P}_{1}$ the pressure indicated by the pump(s) first removed from the leak, and $\mathbf{P}_{\mathrm{k}}$ the pressure at the pump $k$ th removed from the leak.

Case I

Using (1), and treating the vacuum system at the leak as a "current divider network", the following is determined:

$$
\begin{aligned}
\mathbf{P}_{1} & =\mathbf{P}_{0} \frac{\left(\mathbf{c}_{\mathrm{C}}-\Sigma \mathbf{s}\right)}{\mathbf{c}_{\mathrm{C}}} \\
\mathbf{P}_{2} & =\mathbf{P}_{1} \frac{\left(\mathbf{s}_{\mathrm{C}}+\mathbf{c}_{\mathrm{C}}\right)}{\mathrm{c}_{\mathrm{C}}}-\mathrm{P}_{0} \frac{\Sigma \mathbf{S}}{\mathrm{c}_{\mathrm{C}}}
\end{aligned}
$$

After solving for $\mathrm{P}_{2}$, the pressure gradient may be expressed by:

$$
\mathbf{P}_{k}=\mathbf{P}_{\mathrm{k}-1} \frac{\left(\mathbf{S}_{\mathrm{C}}+2 \mathbf{C}_{\mathrm{C}}\right)}{\mathbf{c}_{\mathrm{C}}}-\mathbf{P}_{\mathrm{k}-2}
$$

Note that (1) - (4) give pressures at the AGMCs close to the pumps, rather than at the pumps. However, indicated pressures at the pumps will be directly proportional to the values given by each of these equations. 


\section{Case II}

In this case, conductance of the manifold between the leaking pump and the rest of the AGS must be taken into account. This leads to:

$$
\mathbf{P}_{1}=\mathbf{P}_{\mathrm{p}} \frac{\mathrm{c}^{\prime}\left(\mathrm{C}_{\mathrm{C}}-\Sigma \mathbf{S}\right)}{\mathrm{C}_{\mathrm{C}}\left(\mathrm{C}^{\prime}+\Sigma \mathbf{S}\right)}
$$

where $\quad \mathbf{P}_{\mathrm{p}}=$ the pressure indicated at the pump,

and $\quad \mathbf{c}^{\prime}=$ the conductance between the chamber and the pump.

Lastly, $\quad \mathbf{P}_{\mathrm{k}}=\mathbf{P}_{\mathrm{k}-1} \frac{\left(\mathbf{S}_{\mathrm{C}}+2 \mathbf{C}_{\mathrm{C}}\right)}{\mathbf{C}_{\mathrm{C}}}-\mathbf{P}_{\mathrm{k}-1}$

\section{Case III}

Contrary to intuition, solution of Case III is trivial, and results in a gradient which will be less than noted in either Cases I or II. The pump is a source of outgassing as a consequence of internal heating. However, we claimed (as a worst case) that the net speed was zero. Therefore, the pressure in the pump must be the same as the pressure at the AGMC. Therefore,

$$
\mathbf{P}_{1}=\mathbf{P}_{0} \mathbf{C}_{\mathrm{C}} /\left(\mathbf{C}_{\mathrm{C}}+\mathbf{s}_{\mathrm{C}}\right)
$$

\section{CHARACTERISTICS OF THE AGS}

Values given used for the various parameters are as follows:

$$
\begin{aligned}
& \mathbf{S}_{\mathbf{C}}=64.0 \mathrm{l} / \mathrm{sec} . \\
& \mathbf{C}_{\mathbf{C}}=130.0 \mathrm{sec} . \\
& \Sigma \mathbf{S}=64.66436 \ell / \mathrm{sec}^{\prime}, \\
& \mathbf{C}^{\prime}=92.0 \mathrm{sec} .
\end{aligned}
$$

Normalized results of Cases I \& $\Pi$ are given in Figures $1 \&$ 2, respectively. 


\section{ADDITIONAL CAUSES OF ERRONEOUS PRESSURE INDICATIONS}

Leakage current in either a high voltage cable, the connector or within the sputter-ion pump will cause erroneously high pressure indications for that given pump. EMI in the vicinity of certain rf stations has produced erroneous gauge and pump pressure readings. EMI may also be produced by the "Fourier content" of the beam. Lastly, special beam components used for beam injection and extraction may be causing EMI problems.

Examples of such spurious readings, some of which are caused by EMI, are given in Figures 3 and 4. These are plots of indicated pressures in the DE, E and EF vacuum sectors. Figure 3 is a sampling of pressure data taken with the pump at E-20 turned on. Figure 4 is a sampling of data in a different time interval, and at all times with the pump at E-20 turned off.

Spurious signals are noted at pumps E-15, E-18 and E-20. These reading were taken during a "proton run". In Figure 4 the pressure at E-20 varied between the indicated extremes even though the pump was turned off. In general, the indicated pressures from E-15 through F-3 appear erroneous.

Pressures within the interval D-14 through E-3 also seem to be translated in amplitude $\mathrm{X} 10$ with respect to the interval E-4 through E-14. This may be indicative of a power supply gauge card calibration problem with one of the two respective groups as well as EMI problems in the former group. The pressure "bump" in the interval between E-6 and E-14 is real, and is caused by outgassing of the IPM located at E-10.

During SEB operation, use of the "gauss clock" to block signal sampling, during the early stages of a proton pulse, may cause most of the present EMI problems to disappear. However, FEB operation may result in a whole new set of EMI problems.

\section{REFERENCES:}

1) Guthrie, A., Wakerling, R.K. Vacuum Equipment and Techniques, (McGraw-Hill Book Company, Inc., 1949), p 16.

2) Haruo, H., et al..," Matrix Calculations of Pressures in High-Vacuum Systems," J. Vac. Sci Technol, A 6, 2865 (1988). 
Figure 1. Case I, A Leak In the AGMC Flange.

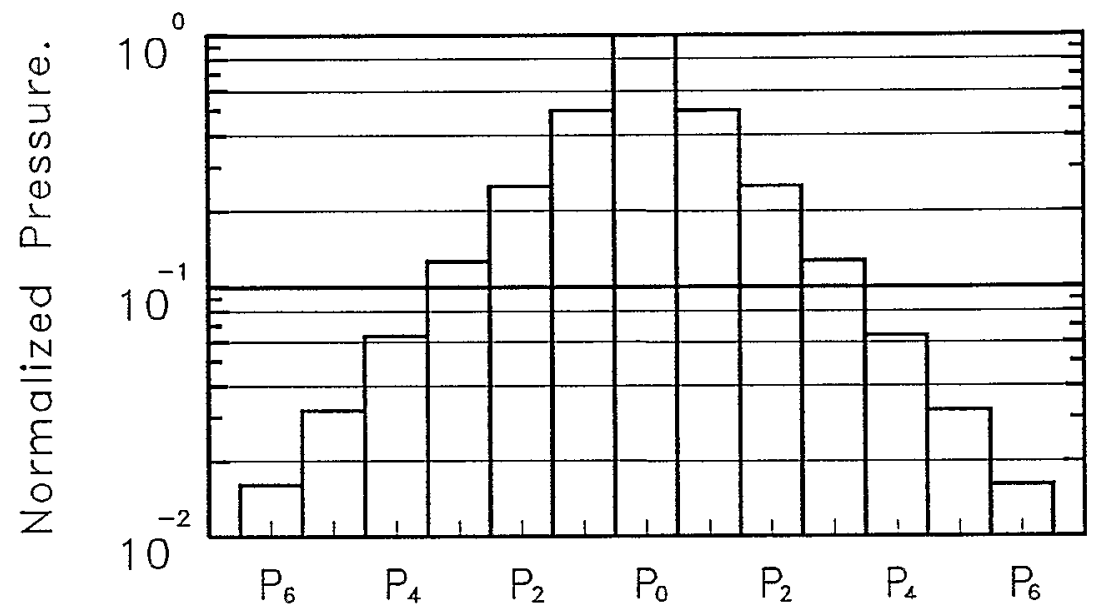

Pumps Up \& Downstream of the Flange Leak.

Figure 2. Case II, A leak In A Pump Body.

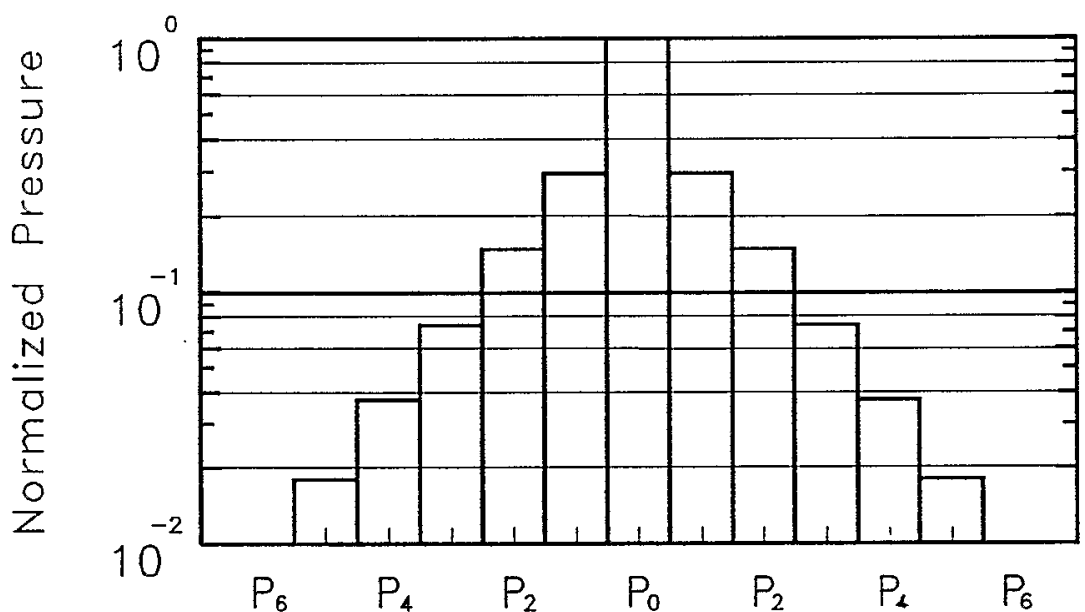

Pumps Up \& Downstream of the Pump Leak. 
Figure 3. Indicated Pressures In Vacuum Sectors D-14

Through F-3 With the Pump at E-20 Turned On.

\section{AGS_VAC groups 11:DE thru 13:EF Fri Feb 3 15:51:32 1989 16 Fri Feb 3 15:53:31 1989}

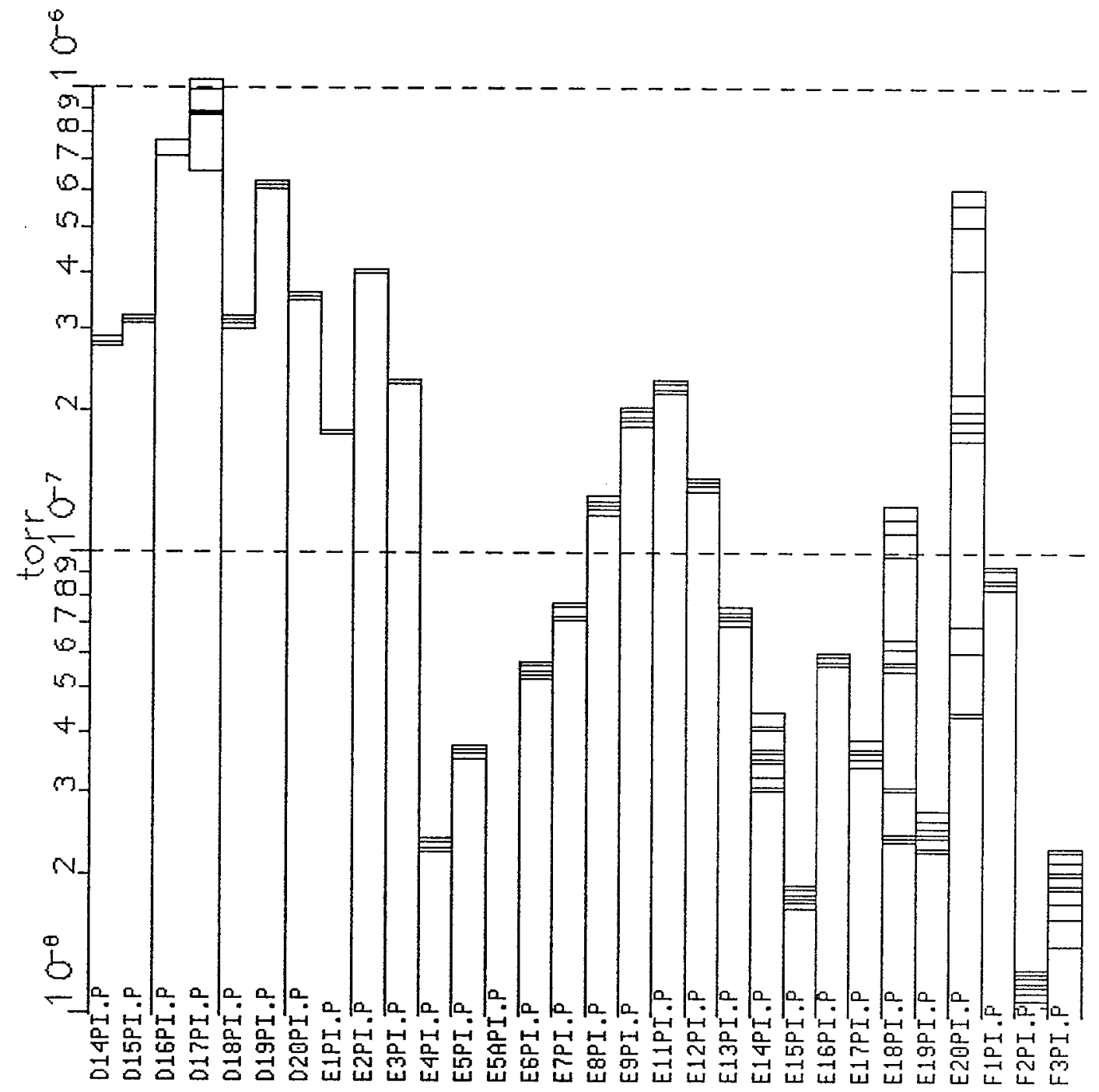


Figure 4. Indicated Pressures In Vacuum Sectors D-14

Through F-3 With the Pump at E-20 Turned Off.

\section{AGS_VAC groups 11:DE thru 13:EF \\ Fri Feb 3 15:56:35 1989 12 Fri Feb 3 15:58:08 1989}

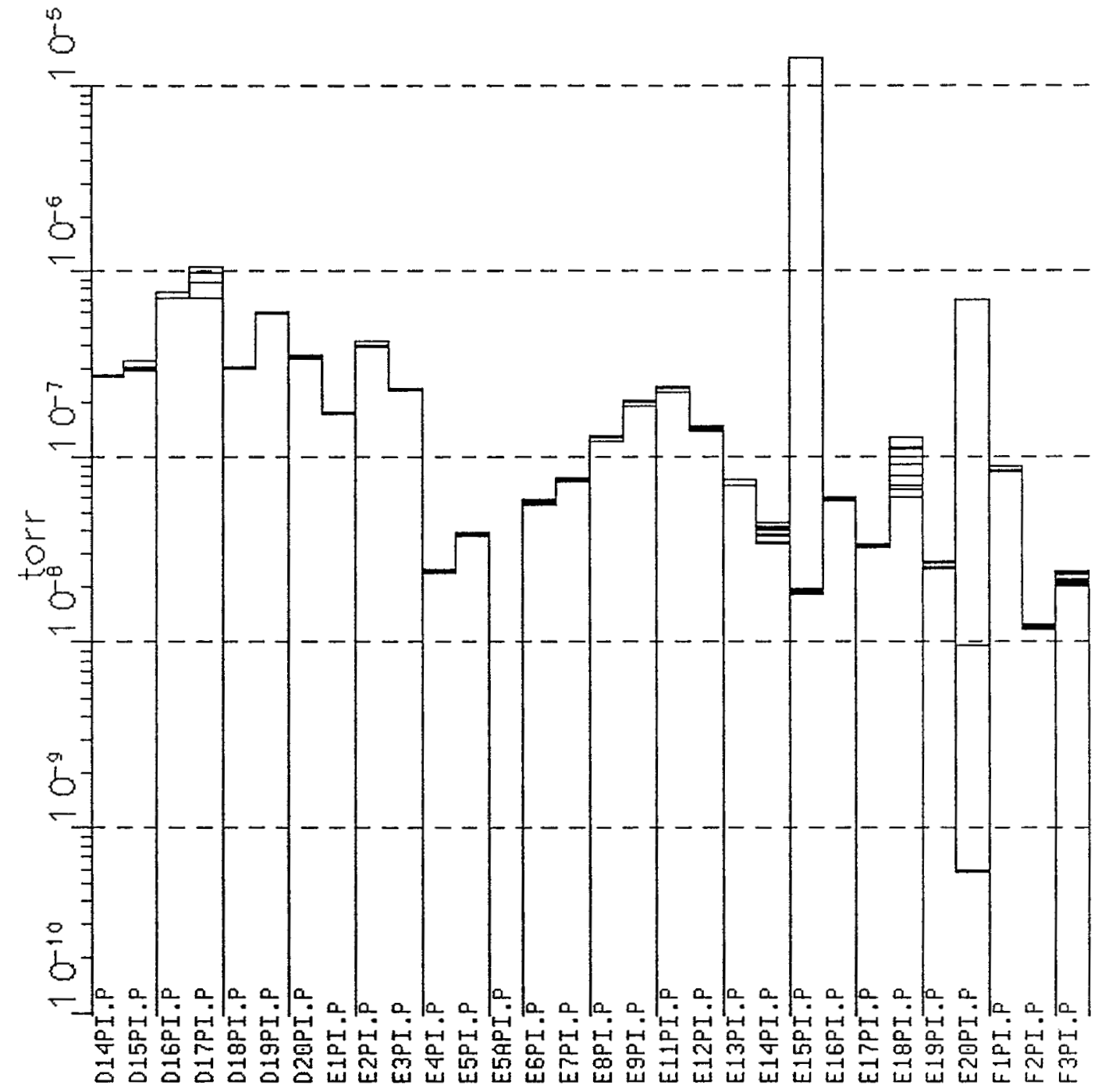

